\title{
Elevated growth differentiating factor 15 expression predicts long-term benefit of docetaxel, cisplatin and 5-fluorouracil induction chemotherapy in patients with oral cancer
}

\author{
XIAO TANG ${ }^{1 *}$, YONG-JIE HU ${ }^{1 *}$, WU-TONG JU ${ }^{1}$, YONG FU ${ }^{1}$, WEN-WEN SUN ${ }^{1}$, YING LIU ${ }^{1}$, YI-RAN TAN ${ }^{1}$, \\ LI-ZHEN WANG ${ }^{2}$, JIANG LI ${ }^{2}$, YAO-YAO TU ${ }^{1}$, CHEN-PING ZHANG ${ }^{1}$, ZHI-YUAN ZHANG ${ }^{1}$ and LAI-PING ZHONG ${ }^{1}$ \\ Departments of ${ }^{1}$ Oral and Maxillofacial-Head and Neck Oncology, and ${ }^{2}$ Oral Pathology, Ninth People's Hospital, \\ Shanghai Jiao Tong University School of Medicine, Shanghai 200011, P.R. China
}

Received April 4, 2017; Accepted February 7, 2018

DOI: $10.3892 / \mathrm{ol} .2018 .8324$

\begin{abstract}
Our previous phase 3 trial (NCT01542931) failed to demonstrate improved survival when docetaxel, cisplatin and 5-fluorouracil (TPF) induction chemotherapy was introduced prior to surgery and postoperative radiotherapy in patients with locally advanced oral squamous cell carcinoma (OSCC). The aim of the present study was to investigate the long-term predictive value of GDF15 expression for potential personalized treatment strategies in OSCC. A total of 256 patients with stage III/IVA OSCC from our phase 3 trial were enrolled in the present study. Immunohistochemical staining against GDF15 was performed in the biopsy samples from 230/256 patients. Kaplan-Meier analysis, followed by the log-rank test, and the Cox proportional hazards model were used for outcome analysis using the statistical SPSS 18.0 software package for Windows. Among the 230 patients, low GDF15 expression was detected in 68 patients and high GDF15 expression was detected in 162 patients. With a median follow-up period of 67 months, the patients with low GDF15 expression exhibited a higher survival rate than those with high GDF15 expression, including 5-year overall survival (73.4 vs. 57.7\%; $\mathrm{P}=0.059$ ), 5-year disease-free survival (64.5 vs. 49.2\%; $\mathrm{P}=0.033$ ), 5-year locoregional recurrence-free survival (66.0 vs. 51.5\%; $\mathrm{P}=0.043$ ) and 5-year distant metastasis-free survival (73.4 vs. $56.6 \%$; $\mathrm{P}=0.038$ ) rates. Furthermore, the cT3/4N0M0 patients with high
\end{abstract}

Correspondence to: Professor Lai-Ping Zhong or Dr Zhi-Yuan Zhang, Department of Oral and Maxillofacial-Head and Neck Oncology, Ninth People's Hospital, Shanghai Jiao Tong University School of Medicine, 639 Zhizaoju Road, Shanghai 200011, P.R. China

E-mail: zhonglp@hotmail.com

E-mail: zhang.z.y@hotmail.com

*Contributed equally

Key words: growth differentiation factor 15, oral squamous cell carcinoma, induction chemotherapy, predictive biomarker
GDF15 expression benefited significantly from TPF induction chemotherapy, including overall survival $(\mathrm{HR}=0.233 ; \mathrm{P}=0.02)$, disease-free survival $(\mathrm{HR}=0.296 ; \mathrm{P}=0.014)$, locoregional recurrence-free survival $(\mathrm{HR}=0.347 ; \mathrm{P}=0.035)$ and distant metastasis-free survival $(\mathrm{HR}=0.212 ; \mathrm{P}=0.013)$ rates. The results of the present study suggested that elevated GDF15 expression may be used as a long-term prognostic biomarker for poor clinical outcomes in patients with locally advanced OSCC. Elevated GDF15 expression in cT3/4N0M0 patients predicts significant long-term benefit of survival from TPF induction chemotherapy.

\section{Introduction}

Oral squamous cell carcinoma (OSCC) is the most common type of cancer in the oral and maxillofacial region. Although substantial efforts have been made to improve the diagnosis and treatment of patients with OSCC, the clinical outcome of patients with OSCC remains poor, with a 5-year survival rate $50-60 \%$, and is even lower in patients with locally advanced lesions $(1,2)$. At present, the recommended treatment strategies for patients with resectable OSCC at late clinical stages are surgery and post-operative radiotherapy or chemoradiotherapy (3).

The aim of induction chemotherapy is to reduce or downstage locally advanced or aggressive tumors, in order to improve the likelihood of primary lesion eradication and to preserve the organs (4). At present, the role of induction chemotherapy in OSCC management remains unclear.

Previously, we conducted a phase 3 trial of TPF (docetaxel, cisplatin and 5-fluorouracil; registration ID, NCT01542931) induction chemotherapy in resectable locally advanced OSCC (5). However, neither short-term nor long-term results from the initial trial revealed improvement in clinical outcomes. Only a proportion of the patients benefited from induction chemotherapy. It is considered that there may be a specific patient population defined by clinical and/or molecular criteria that would benefit from induction chemotherapy $(5,6)$. Therefore, identifying candidate biomarkers to predict the response to a particular treatment strategy may be useful in improving overall survival (OS). 
Growth differentiation factor 15 (GDF15) is a member of transforming growth factor- $\beta$ superfamily, involved in a wide variety of physiological and pathological processes $(7,8)$. Although the function of GDF15 in oncogenesis remains unclear, overexpression of GDF15 has been reported in prostate, colon, pancreas, thyroid and breast carcinomas, as well as in OSCC (9-12). Additionally, an elevated serum GDF15 concentration has been reported to be associated with disease progression, shorter survival times and recurrence (7,13-18). In our previous studies, elevated GDF15 mRNA and protein expression was correlated with the malignancy of OSCC tissues (12), and the patients with low GDF15 expression exhibited a 3-year survival advantage (19). It is likely that GDF15 expression may be a candidate prognostic/predictive biomarker for patients with OSCC.

The present study identified the long-term prognostic value of GDF15 expression in patients with stage III/IVA OSCC, as well as its long-term predictive value for TPF induction chemotherapy, compared with the standard treatment alone in the phase 3 trial.

\section{Materials and methods}

Patients. A total of 256 patients (179 men and 77 women; aged from 26 to 75 years, with a mean of 55.4 years) with resectable stage III and IVA OSCC (T1-2N1-2M0 or T3-4N0-2M0) using the Tumor-Node-Metastasis staging system (20), who came from a prospective, randomized, phase 3 trial at Ninth People's Hospital, Shanghai Jiao Tong University School of Medicine (Shanghai, China; registration ID: NCT01542931), were enrolled in the present study. The aim of the trial was to test the hypothesis that TPF induction chemotherapy administered prior to surgery and post-operative radiotherapy improves survival in patients with locally advanced OSCC. The experimental group received TPF induction chemotherapy followed by surgery and post-operative radiotherapy; the control group underwent surgery and post-operative radiotherapy; and there were 128 patients in each group. The detailed treatment protocol was as previously described (5). In patients assigned to the experimental group, the palpable edges of the primary lesion (both the longest and shortest axis) were marked prior induction chemotherapy by at least four points that were $0.5 \mathrm{~cm}$ away from the lesion. Chemotherapy consisted of docetaxel $\left(75 \mathrm{mg} / \mathrm{m}^{2}\right)$, cisplatin $\left(75 \mathrm{mg} / \mathrm{m}^{2}\right)$ and 5 -fluorouracil ( $750 \mathrm{mg} / \mathrm{m}^{2} /$ day) as a 120 -h infusion for 5 days. Induction chemotherapy was administered every 3 weeks for 2 cycles. Surgery was performed $\geq 2$ weeks after completion of induction chemotherapy. Radical resection of the primary lesion and full neck dissection with appropriate reconstruction was performed. The safety margins of the primary lesion were $1.5 \mathrm{~cm}$ away from the palpable margins; for patients who received induction chemotherapy, the safety margins were $1.0 \mathrm{~cm}$ away from the marks that had been placed prior to induction chemotherapy. Frozen sections of the surgical margins, including one or more of the anterior, posterior, upper and lower margins, which are at the mucosal surfaces and bottom margins, which are the deepest muscle layers, towards the muscle layers of the surgical bed, which is the remaining surface following tumor removal, were removed during the surgery. If cancer cells were identified in the margins, wider resection for at least $1.0 \mathrm{~cm}$ away from the positive margins was performed as possible as the surgeons can. Frozen section of the new surgical margins would be performed again to ensure the margins to be negative. Post-operative radiotherapy was initiated 4-6 weeks after surgery. Standard conformal or intensity-modulated radiotherapy was utilized, at a dose of 1.8-2 Gy/day, 5 days/week for 6 weeks, totaling 54-60 Gy. In patients with high-risk features (defined if two or more regional lymph nodes were involved, if there was an extracapsular spread of disease, or a microscopically involved mucosal margin of resection), a total dose of $66 \mathrm{~Gy}$ was recommended.

Immunohistochemistry. Formalin-fixed (at room temperature for $12-18 \mathrm{~h}$ for $5 \mu \mathrm{m}$ sections) and paraffin-embedded biopsies were collected for immunohistochemical staining against GDF15. The methodology and assessment were as described previously (19). Following deparaffinization with xylene, the sections $(5 \mu \mathrm{m})$ were rehydrated using an ethanol series of 100 , 95,85 and $5 \%$ ethanol, then distilled water. Prior to incubation with antibody solutions, the sections were heated by a water bath at $98^{\circ} \mathrm{C}$ with $0.01 \mathrm{M}$ citrate buffer solution $(\mathrm{pH}=6.0)$ for $20 \mathrm{~min}$ for antigen retrieval and cooled at room temperature, then washed with phosphate buffer solution 3 times for $5 \mathrm{~min}$ each. Then, the sections were incubated with the primary rabbit polyclonal antibody against GDF15 (cat no. ab82569; dilution, 1:100; Abcam, Cambridge, UK) overnight at $4^{\circ} \mathrm{C}$ and the secondary goat polyclonal to rabbit $\operatorname{IgG}$ antibody for $1 \mathrm{~h}$ at room temperature (cat no. ab150077; dilution, 1:3,000; Abcam), visualized using a 3,3'-diaminobenzidine detection kit (cat no. K067311; Dako; Agilent Technologies, Inc., Santa Clara, CA, USA). Positive staining for GDF15 expression was observed in the cytoplasm. Two pathologists performed blind examination using a light microscope at a magnification of $\mathrm{x} 200$. The GDF15 expression level was determined using the immunoreactive score (IRS) system, including a proportion score (PS) and an intensity score (IS). The PS was calculated as the percentage ratio of positive GDF15-stained tumor cells to the total number of tumor cells, classified as follows: 0 , $0 \% ; 1,1-10 \% ; 2,11-50 \% ; 3,51-80 \%$; and $4,>80 \%$. The IS was calculated as the staining intensity by visual assessment and was scored as follows: 0 , negative; $1+$, weak; $2+$, moderate; and $3+$, strong. The final GDF15 expression score was calculated using the PS and the IS (IRS=PSxIS), which ranged between 0 and 12. GDF15 expression was classified as low when IRS $\leq 3$ and as high when IRS $\geq 4$ (Fig. 1) $(19,21)$.

Follow-up and outcomes. Following initial treatment, patients were monitored every 3 months in the first 2 years, every 6 months in the subsequent 3-5 years, and once a year thereafter until mortality or data censoring. OS was counted from the date of random assignment to the date of mortality. Disease-free survival (DFS), locoregional recurrence-free survival (LRFS) and distant metastasis-free survival (DMFS) were counted from the date of random assignment to the date of recurrence, locoregional recurrence, distant metastasis or mortality, respectively.

Statistical analysis. For descriptive analysis, categorical data are expressed as the number and percentage. Survival analyses was conducted using the Kaplan-Meier method, followed by 


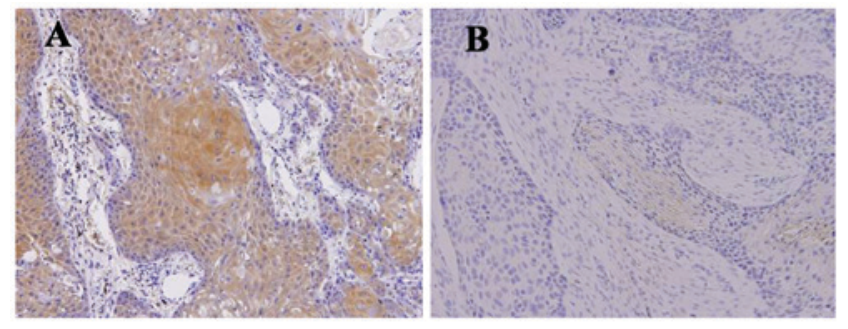

Figure 1. GDF15 expression in OSCC biopsy samples. (A) High and (B) low GDF15 expression in OSCC biopsy samples. Magnification, x200. GDF15, growth differentiation factor 15 ; OSCC, oral squamous cell carcinoma.

the log-rank test. Hazard ratios (HR) were calculated using the Cox proportional hazards model. Subgroup survival analyses according to the baseline characteristics (including the following subgroups: Sex, male and female; age, $<60$ years and $\geq 60$ years; site, tongue and non-tongue; $\mathrm{T}$ stage, $\mathrm{cT} 1 / 2 \mathrm{~N} 1-2 \mathrm{M} 0$ and cT3/4N0-2M0; N stage, cT3/T4N0M0 and cT1-4N1-2M0; clinical stage, clinical III and IV; pathological differentiation grade, well and moderately/poorly (22); smoke status, never smokers and current/former smokers; alcohol use, positive and negative use) were performed in patients with low or high GDF15 expression. All hypothesis-generating tests were two-sided and $\mathrm{P}<0.05$ was considered to indicate a statistically significant difference. Data analyses were performed using the statistical software SPSS 18.0 for Windows (SPSS, Inc., Chicago, IL, USA).

\section{Results}

Patient characteristics and long-term treatment outcomes. Among the 256 patients, pretreatment biopsy samples were available from 230 patients for assessing GDF15 expression, including 104 patients in the experiment group and 126 patients in the control group. The difference in distribution of baseline characteristics, including sex, age, primary tumor site, clinical Tumor-Node-Metastasis stage or pathological differentiation grade between the patients with low and high GDF15 expression was not statistically significant $(\mathrm{P}>0.05)$ (19). At the time of data cut-off in December 2014, the median follow-up period was 67 months. Although patients in the experimental group had a slight survival advantage in OS, DFS, LRFS and DMFS, this difference was not significant (Fig. 2).

Low GDF15 expression indicates better long-term outcomes in patients with OSCC. Among the 230 patients with OSCC with biopsy samples, low GDF15 expression was observed in 68 patients (31 in the experimental group and 37 in the control group) and high GDF15 expression in 162 patients (73 in the experimental group and 89 in the control group), with similar distribution of GDF15 expression between the two groups $(\mathrm{P}=0.942)$.

In the patients with low GDF15 expression, the 5-year OS, DFS, LRFS and DMFS rates were 73.4, 64.5, 66.0 and $73.4 \%$, respectively, the locoregional recurrence rate was $27.9 \%$ and the distant metastasis rate was $5.9 \%$. In the patients with high GDF15 expression, the 5-year OS, DFS, LRFS and DMFS rates were $57.7,49.2,51.5$ and $56.6 \%$, respectively,

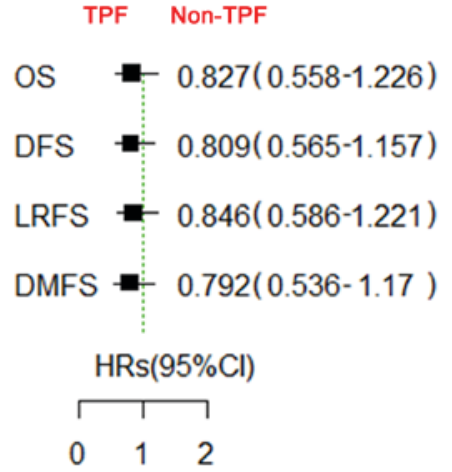

Figure 2. Survival comparison between the patients treated with and without TPF induction chemotherapy. In general, the patients did not benefit from TPF induction chemotherapy when added prior to standard surgery and postoperative radiotherapy. TPF, docetaxel, cisplatin and 5-fluorouracil; GDF15, growth differentiation factor 15; OS, overall survival; DFS, disease-free survival; LRFS, locoregional recurrence-free survival; DMFS, distant metastasis-free survival; HR, hazards ratio; $\mathrm{CI}$, confidence interval. The green line is a reference line for $\mathrm{HR}=1$.

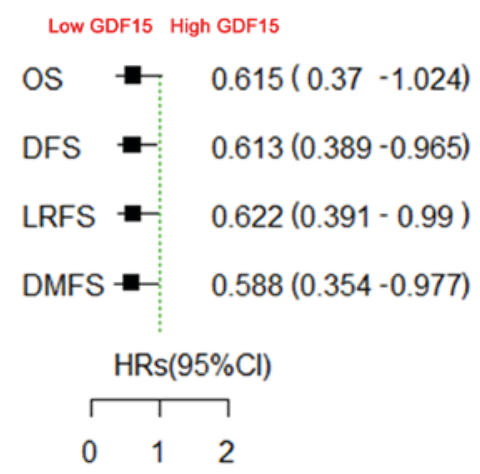

Figure 3. Prognostic comparison between the patients with low and high GDF15 expression. The patients with low GDF15 expression exhibited better clinical outcomes than those with high GDF15 expression. TPF, docetaxel, cisplatin and 5-fluorouracil; GDF15, growth differentiation factor 15; OS, overall survival; DFS, disease-free survival; LRFS, locoregional recurrence-free survival; DMFS, distant metastasis-free survival; $\mathrm{HR}$, hazards ratio; CI, confidence interval. The green line is a reference line for $H R=1$.

the locoregional recurrence rate was $39.5 \%$ and the distant metastasis rate was $9.3 \%$

Patients with low GDF15 expression exhibited significantly better long-term outcomes, with regards to DFS ( $\mathrm{P}=0.033)$, LRFS $(\mathrm{P}=0.043)$ and DMFS $(\mathrm{P}=0.038)$ rates, compared with those with high GDF15 expression. Although the difference in the OS rate was not significant, there was a trend towards a better OS rate $(\mathrm{P}=0.059)$ for patients with low GDF15 expression than those with high GDF15 expression (Fig. 3).

Analysis of the impact of baseline characteristics on the time-to-event end points was performed using a univariate Cox proportional hazards model according to the method used previously $(19,23)$. Risk factors for OS, DFS, LRFS and DMFS included GDF15 expression (low vs. high), lymph node status (cN- vs. $\mathrm{cN}+$ ) and clinical stage (stage III vs. stage IV). The risk factors for GDF15 expression and clinical stage were used in subsequent multivariate Cox model analysis, lymph node status was not included due to the direct association between clinical stage and lymph node status. GDF15 expression and 
clinical stage were independent risk factors for OS $(\mathrm{P}=0.022$ and $\mathrm{P}<0.001)$, DFS $(\mathrm{P}=0.01$ and $\mathrm{P}<0.001)$, LRFS $(\mathrm{P}=0.012$ and $\mathrm{P}<0.001)$ and DMFS $(\mathrm{P}=0.014$ and $\mathrm{P}<0.001)$ rates.

High GDF15 expression predicts significant long-term survival benefit from TPF induction chemotherapy in patients with $c T 3 / 4 N O M O O S C C$. In order to analyze whether the patients with low or high GDF15 expression may benefit from TPF induction chemotherapy, survival analysis was performed to determine the difference in the outcomes of patients with different expression levels of GDF15 and with differing characteristics. In general, the patients did not benefit from TPF induction chemotherapy, neither those with low GDF15 expression nor those with high GDF15 expression (Fig. 4). Subgroup survival analysis according to baseline characteristics revealed that only the patients with high GDF15 expression and $\mathrm{cN} 0$ benefited from TPF induction chemotherapy with respect to $\mathrm{OS}(\mathrm{HR}=0.233 ; 95 \% \mathrm{CI}=0.068-0.795$; $\mathrm{P}=0.02)$, DFS (HR=0.296; 95\% CI $=0.111-0.785 ; \mathrm{P}=0.014)$, LRFS (HR=0.347; 95\% CI=0.129-0.929; $\mathrm{P}=0.035)$ and DMFS (HR=0.212; 95\% CI=0.062-0.72; $\mathrm{P}=0.013$; Fig. 5); while the patients with low GDF15 expression and cN0 did not benefit from TPF induction chemotherapy. No significant difference was identified between any other subgroups.

\section{Discussion}

The present study revealed that elevated GDF15 expression may be used as a long-term prognostic biomarker for poor clinical outcomes in patients with locally advanced OSCC. Elevated GDF15 expression in patients with cT3/4N0M0 disease predicted significant long-term benefit of survival from the addition of TPF induction chemotherapy prior to standard treatment of surgery and post-operative radiotherapy, compared with the standard treatment alone in OSCC. The results of the present study suggested a personalized treatment regimen in which patients with cT3/4NOM0 disease exhibiting high GDF15 expression would receive TPF induction chemotherapy prior to surgery for long-term benefit of clinical outcomes while the others would not receive TPF induction chemotherapy prior to surgery, a situation that would avoid the excessive toxicities of chemotherapy and the delay of definitive treatment. As such, we previously designed and performed a randomized clinical study of TPF induction chemotherapy in patients with cT3/4N0M0 OSCC, prospectively embedding GDF15 expression as a predictive biomarker (registration ID, NCT02285530).

GDF15 is involved in the regulation of various physiological and pathological processes, including inflammation, cellular stress, immune response, tissue repair and oncogenesis $(7,8)$. Elevated GDF15 expression has been reported to be correlated with neoplasm progression in several types of cancer, including prostate, colon, pancreas, thyroid and breast carcinomas (9-11). In our previous study, elevated GDF15 expression was revealed to promote the tumorigenesis and progression of OSCC through Akt and extracellular signal-regulated kinase 1/2 phosphorylation (12). In the present study with long-term follow-up, the patients with OSCC exhibiting elevated GDF15 expression would have a poorer prognosis than those with low GDF15 expression. This is similar to other types of cancer, including prostate, ovarian and colorectal cancers $(10,24,25)$.

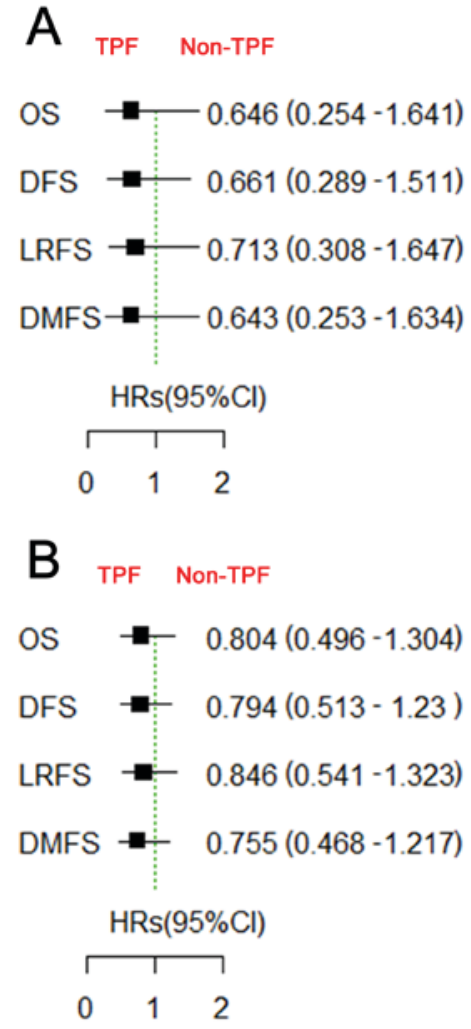

Figure 4. Survival comparison between patients treated with and without TPF induction chemotherapy, based on GDF15 expression. Patients with (A) low or (B) high GDF15 expression did not benefit from TPF induction chemotherapy. TPF, docetaxel, cisplatin and 5-fluorouracil; GDF15, growth differentiation factor 15; OS, overall survival; DFS, disease-free survival; LRFS, locoregional recurrence-free survival; DMFS, distant metastasis-free survival; HR, hazards ratio; CI, confidence interval.

However, to the best of our knowledge, the present study is the first to report the long-term results of GDF15 expression as a predictive biomarker for survival benefit from TPF induction chemotherapy in patients with OSCC. The patients with cT3/4N0M0 disease exhibiting high GDF15 expression benefited from TPF induction chemotherapy with respect to OS, DFS, LRFS and DMFS; while the other patients did not benefit from TPF induction chemotherapy.

Although the detailed beneficial mechanism of TPF inductive chemotherapy agents on the patients with cT3/4N0M0 disease exhibiting high GDF15 expression with regards to long-term outcomes, potential associations between GDF15 expression and chemotherapy agents have been investigated with inconsistent results. For example, in colorectal cancer, GDF15 knockdown with small interfering RNA has been reported to prevent docetaxel-induced cell death in the wild-type p53 HCT-116 cell line (26). By contrast, GDF15 silencing prior to application of oxaliplatin and 5-fluorouracil has also been reported to sensitize wild-type p53 colorectal cancer cells to drug-induced apoptosis (27). In prostate cancer, overexpression of GDF15 has been reported to be associated with resistance to docetaxel, which suggests the potential utility of GDF15 as a predictive biomarker of response to docetaxel in prostate cancer $(10,28,29)$. However, in the present study, the patients with OSCC exhibiting a high GDF15 expression had a poorer prognosis compared with those exhibiting a low GDF15 expression. The subgroup patients with large tumor size only 

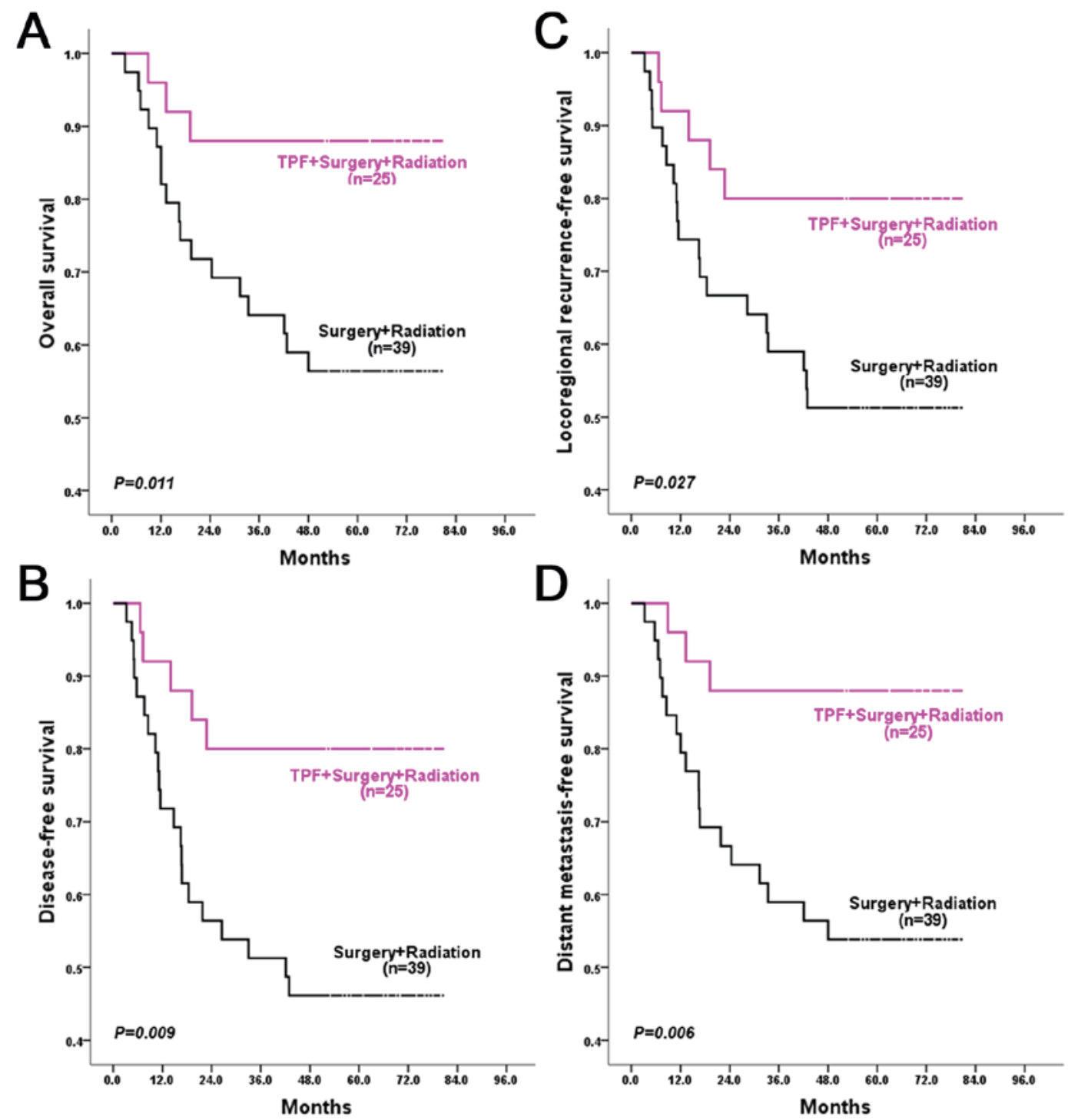

Figure 5. Prognostic comparison between the patients with cT3/4NOM0 disease exhibiting high GDF15 expression, who were treated with and without TPF induction chemotherapy. The patients with cT3/4N0M0 disease exhibiting high GDF15 expression benefited from TPF induction chemotherapy with regard to (A) overall survival, (B) disease-free survival, (C) locoregional recurrence-free survival and (D) distant metastasis-free survival. TPF, docetaxel, cisplatin and 5-fluorouracil; GDF15, growth differentiation factor 15.

(cT3/4NOM0) and high GDF15 expression benefited from TPF induction chemotherapy, indicating that the patients with OSCC overexpressing GDF15 may be sensitive to docetaxel, cisplatin and 5-fluorouracil. It may be possible to control local primary tumors with GDF15 overexpression using TPF induction chemotherapy agents. However, when lymph node metastasis occurs in those patients, induction chemotherapy agents may be unable to control the disease. It has been reported by previous clinical trials that the patients with head neck squamous cell carcinoma and advanced lymph node metastasis do not benefit from TPF induction chemotherapy (30-32). Therefore, induction chemotherapy may be unable to control the patients with lymph node metastasis. However, further studies are required to reveal the detailed mechanisms of this.

The results of the present study suggested that elevated GDF15 expression may be used as a long-term prognostic biomarker for poor clinical outcomes in patients with locally advanced OSCC. Elevated GDF15 expression in patients with cT3/4N0M0 disease predicted significant long-term benefit of survival from the addition of TPF induction chemotherapy ahead of standard treatment of surgery and post-operative radiotherapy, compared with the standard treatment alone in OSCC. Detailed mechanism studies are required to explain the clinical phenomenon and to provide basic support to optimize the personalized treatment strategies in OSCC.

\section{Acknowledgements}

Not applicable.

\section{Funding}

The present study was supported by the National Natural Science Foundation of China (grant nos. 81672660 and 81472519), the Shuguang Program of Shanghai Municipal Education Commission (grant no. 17SG18) and by the Shanghai Jiao Tong University School of Medicine (grant no. 15ZH2008 and 17XJ12004). 


\section{Availability of data and materials}

The data and materials are available from the corresponding authors if necessary.

\section{Authors' contributions}

$\mathrm{LZ}$ and $\mathrm{ZZ}$ were responsible for the study design, interpretation of the data and revision of the manuscript. XT and YH were responsible for data acquisition, analysis of the work presented and the preparation of the manuscript. WJ, YF, WS, YL, YT, LW, JL, YT and CZ participated in the clinical management of the patients. All authors read and approved the final manuscript.

\section{Ethics approval and consent to participate}

The present study was approved by the Institutional Review Board of Ninth People's Hospital, Shanghai Jiao Tong University School of Medicine (IRB approval documents of 2008-12 and 2014-75) and written informed consent was obtained from all participants.

\section{Consent for publication}

Written informed consent was obtained from all participants.

\section{Competing interests}

The authors declare that they have no competing interests.

\section{References}

1. Torre LA, Bray F, Siegel RL, Ferlay J, Lortet-Tieulent J and Jemal A: Global cancer statistics, 2012. CA Cancer J Clin 65: 87-108, 2015.

2. Leemans CR, Braakhuis BJ and Brakenhoff RH: The molecular biology of head and neck cancer. Nat Rev Cancer 11: 9-22, 2011

3. National Comprehensive Cancer Network: NCCN Clinical Practice Guidelines in Oncology (NCCN Guidelines ${ }^{\circledR}$ ) Head and Neck Cancers (Version 1.2016). http://www.ncen.org/professionals/physician_gls/pdf/head-and-neck.pdf. Accessed August 5, 2016

4. Zhong LP and Zhang ZY: Reply to K. Devisetty et al. J Clin Oncol 31: 2972-2973, 2013.

5. Zhong LP, Zhang CP, Ren GX, Guo W, William WN Jr, Sun J, Zhu HG, Tu WY, Li J, Cai YL, et al: Randomized phase III trial of induction chemotherapy with docetaxel, cisplatin, and fluorouracil followed by surgery versus up-front surgery in locally advanced resectable oral squamous cell carcinoma. J Clin Oncol 31: 744-751, 2013.

6. Zhong LP, Zhang CP, Ren GX, Guo W, William WN Jr, Hong CS, Sun J, Zhu HG, Tu WY, Li J, et al: Long-term results of a randomized phase III trial of TPF induction chemotherapy followed by surgery and radiotherapy in locally advanced oral squamous cell carcinoma. Oncotarget 6: 18707-18714, 2015.

7. Breit SN, Johnen H, Cook AD, Tsai VW, Mohammad MG, Kuffner T, Zhang HP, Marquis CP, Jiang L, Lockwood G, et al: The TGF- $\beta$ superfamily cytokine, MIC-1/GDF15: A pleotrophic cytokine with roles in inflammation, cancer and metabolism. Growth Factors 29: 187-195, 2011

8. Corre J, Hebraud B and Bourin P: Concise review: Growth differentiation factor 15 in pathology: A clinical role? Stem Cells Transl Med 2: 946-952, 2013.

9. Khaled YS, Elkord E and Ammori BJ: Macrophage inhibitory cytokine-1: A review of its pleiotropic actions in cancer. Cancer Biomark 11: 183-190, 2012.

10. Brown DA, Lindmark F, Stattin P, Bälter K, Adami HO, Zheng SL, $\mathrm{Xu} \mathrm{J}$, Isaacs WB, Grönberg $\mathrm{H}$, Breit SN and Wiklund FE: Macrophage inhibitory cytokine 1: A new prognostic marker in prostate cancer. Clin Cancer Res 15: 6658-6664, 2009.
11. Wallin U, Glimelius B, Jirstrom K, Darmanis S, Nong RY, Pontén F, Johansson C, Påhlman L and Birgisson H: Growth differentiation factor 15: A prognostic marker for recurrence in colorectal cancer. Br J Cancer 104: 1619-1627, 2011.

12. Zhang L, Yang X, Pan HY, Zhou XJ, Li J, Chen WT, Zhong LP and Zhang ZY: Expression of growth differentiation factor 15 is positively correlated with histopathological malignant grade and in vitro cell proliferation in oral squamous cell carcinoma. Oral Oncol 45: 627-632, 2009.

13. Brown DA, Stephan C, Ward RL, Law M, Hunter M, Bauskin AR, Amin J, Jung K, Diamandis EP, Hampton GM, et al: Measurement of serum levels of macrophage inhibitory cytokine 1 combined with prostate-specific antigen improves prostate cancer diagnosis. Clin Cancer Res 12: 89-96, 2006.

14. Selander KS, Brown DA, Sequeiros GB, Hunter M, Desmond R, Parpala T, Risteli J, Breit SN and Jukkola-Vuorinen A: Serum macrophage inhibitory cytokine-1 concentrations correlate with the presence of prostate cancer bone metastases. Cancer Epidemiol Biomarkers Prev 16: 532-537, 2007.

15. Brown DA, Ward RL, Buckhaults P, Liu T, Romans KE, Hawkins NJ, Bauskin AR, Kinzler KW, Vogelstein B and Breit SN: MIC-1 serum level and genotype: Associations with progress and prognosis of colorectal carcinoma. Clin Cancer Res 9: 2642-2650, 2003.

16. Schiegnitz E, Kämmerer PW, Koch FP, Kruger M, Berres M and Al-Nawas B: GDF 15 as an anti-apoptotic, diagnostic and prognostic marker in oral squamous cell carcinoma. Oral Oncol 48: 608-614, 2012.

17. Schiegnitz E, Kämmerer PW, Rode K, Schorn T, Brieger J and Al-Nawas B: Growth differentiation factor 15 as a radiation-induced marker in oral carcinoma increasing radiation resistance. J Oral Pathol Med 45: 63-69, 2016.

18. Shnaper S, Desbaillets I, Brown DA, Murat A, Migliavacca E, Schluep M, Ostermann S, Hamou MF, Stupp R, Breit SN, et al: Elevated levels of MIC-1/GDF15 in the cerebrospinal fluid of patients are associated with glioblastoma and worse outcome. Int J Cancer 125: 2624-2630, 2009.

19. Yang CZ, Ma J, Zhu DW, Montgomery B, Wang LZ, Li J, Zhang ZY, Zhang CP and Zhong LP: GDF15 is a potential predictive biomarker for TPF induction chemotherapy and promotes tumorigenesis and progression in oral squamous cell carcinoma. Ann Oncol 25: 1215-1222, 2014.

20. Sobin LH and Wittekind $\mathrm{CH}$ (eds): International Union Against Cancer (UICC): TNM classification of malignant tumours. 6th edition. Wiley, New York, NY, 2002.

21. Kaemmerer D, Peter L, Lupp A, Schulz S, Sänger J, Baum RP, Prasad V and Hommann M: Comparing of IRS and Her2 as immunohistochemical scoring schemes in gastroenteropancreatic neuroendocrine tumors. Int J Clin Exp Pathol 5: 187-194, 2012.

22. Arduino PG, Carrozzo M, Chiecchio A, Broccoletti R, Tirone F, Borra E, Bertolusso G and Gandolfo S: Clinical and histopathologic independent prognostic factors in oral squamous cell carcinoma: A retrospective study of 334 cases. J Oral Maxillofac Surg 66: 1570-1579, 2008.

23. Zhong LP, Zhu DW, William WN Jr, Liu Y, Ma J, Yang CZ, Yang X, Wang LZ, Li J, Myers JN, et al: Elevated cyclin D1 expression is predictive for a benefit from TPF induction chemotherapy in oral squamous cell carcinoma patients with advanced nodal disease. Mol Cancer Ther 12: 1112-1121, 2013.

24. Staff AC, Bock AJ, Becker C, Kempf T, Wollert KC and Davidson B: Growth differentiation factor-15 as a prognostic biomarker in ovarian cancer. Gynecol Oncol 118: 237-243, 2010.

25. Zhao L, Lee BY, Brown DA, Molloy MP, Marx GM, Pavlakis N, Boyer MJ, Stockler MR, Kaplan W, Breit SN, et al: Identification of candidate biomarkers of therapeutic response to docetaxel by proteomic profiling. Cancer Res 69: 7696-7703, 2009.

26. Kim IY, Park SY, Kang Y, Thapa D, Choi HG and Kim JA: Role of nonsteroidal anti-inflammatory drug-activated gene-1 in docetaxel-induced cell death of human colorectal cancer cells with different p53 status. Arch Pharm Res 34: 323-330, 2011.

27. Proutski I, Stevenson L, Allen WL, McCulla A, Boyer J, McLean EG, Longley DB and Johnston PG: Prostate-derived factor-a novel inhibitor of drug-induced cell death in colon cancer cells. Mol Cancer Ther 8: 2566-2574, 2009.

28. Magadoux L, Isambert N, Plenchette S, Jeannin JF and Laurens V: Emerging targets to monitor and overcome docetaxel resistance in castration resistant prostate cancer (review). Int J Oncol 45: 919-928, 2014. 
29. Mahon KL, Lin HM, Castillo L, Lee BY, Lee-Ng M, Chatfield MD, Chiam K, Breit SN, Brown DA, Molloy MP, et al: Cytokine profiling of docetaxel-resistant castration-resistant prostate cancer. Br J Cancer 112: 1340-1348, 2015.

30. Hitt R, Grau JJ, López-Pousa A, Berrocal A, García-Girón C, Irigoyen A, Sastre J, Martínez-Trufero J, Brandariz Castelo JA, Verger E, et al: A randomized phase III trial comparing induction chemotherapy followed by chemoradiotherapy versus chemoradiotherapy alone as treatment of unresectable head and neck cancer. Ann Oncol 25: 216-225, 2014.

31. Haddad R, O'Neill A, Rabinowits G, Tishler R, Khuri F, Adkins D, Clark J, Sarlis N, Lorch J, Beitler JJ, et al: Induction chemotherapy followed by concurrent chemoradiotherapy (sequential chemoradiotherapy) versus concurrent chemoradiotherapy alone in locally advanced head and neck cancer (PARADIGM): A randomised phase 3 trial. Lancet Oncol 14: 257-264, 2013.
32. Cohen EE, Karrison TG, Kocherginsky M, Mueller J, Egan R, Huang CH, Brockstein BE, Agulnik MB, Mittal BB, Yunus F, et al: Phase III randomized trial of induction chemotherapy in patients with N2 or N3 locally advanced head and neck cancer. J Clin Oncol 32: 2735-2743, 2014. 